\title{
中国梭梭属植物历史分布格局及其驱动机制
}

张丹 $^{1}$ ，马松梅 ${ }^{*}$, 魏博 ${ }^{1}$, 王春成 ${ }^{1}$, 张林 ${ }^{2}$, 间涵 ${ }^{2}$

1. 石河子大学生命科学学院绿洲城镇与山盆生态系统重点实验室, 新疆石河子 $832000 ; 2$. 石河子大学理学院绿洲城镇与山盆生态系统重 点实验室/干旱区景观生态重点实验室，新疆石河子 832000

摘要: 梭梭属(Haloxylon)植物是藜科的古老子遗物种, 探究末次间冰期(last interglacial period, LIG)和末次盛冰期(last glacial maximum period, LGM) 以来中国梭梭属植物的历史地理分布格局及其驱动机制, 对了解气候变化背景下旱生植物区系的发 展与演化具有重要意义。本研究利用梭梭属 85 个自然分布点数据(60条梭梭(Haloxylon ammodendron)分布记录、25条白梭梭(H. persicum)分布记录)和 2 套环境因子数据, 整合GIS空间分析和 9 种物种分布模型, 分析了梭梭属末次间冰期以来的地理分布格 局变化及其驱动机制。基于 62 个梭梭属种群的叶绿体基因测序数据, 利用最小成本路径方法, 模拟了末次间冰期以来梭梭属 可能的扩散路径。利用R软件prcomp函数对影响梭梭属分布的环境变量进行主成分分析(principal component analysis, PCA), 评价了环境变量对梭梭属适宜分布的贡献, 并分析了关键变量与分布适宜性的相关性。结果表明: (1)集成模型的模拟精度较 单一模型显著提升, 且对白梭梭的模拟精度高于梭梭; (2)末次间冰期以来, 梭梭属植物的分布均经历了显著收缩和冰后期扩 张, 末次间冰期至末次盛冰期时期, 在准噶尔盆地、塔里木盆地西部广泛分布的梭梭大面积向西退缩至避难所(准噶尔盆地西 北缘和塔里木盆地西北缘); 白梭梭从准噶尔盆地、塔里木盆地西端向南退缩至避难所(准噶尔盆地南缘); 末次盛冰期至今, 梭梭向东沿甘肃北部扩张直至内蒙古西部阿拉善荒漠, 白梭梭向东北方向小范围扩张, 占据了准噶尔盆地西部和南缘; (3)末 次间冰期以来的气候波动对梭梭属植物的分布存在较大限制, 降水因子主导了梭梭属适宜分布面积的变化, 温度因子影响了 梭梭属分布适宜性的高低。

关键词: 梭梭属; 历史分布格局; 冰后期扩散路径; 末次盛冰期; 末次间冰期

张丹, 马松梅, 魏博, 王春成, 张林, 阊涵 (2022) 中国梭梭属植物历史分布格局及其驱动机制. 生物多样性, 30, 21192. doi: 10.17520/biods.2021192. Zhang D, Ma SM, Wei B, Wang CC, Zhang L, Yan H (2022) Historical distribution pattern and driving mechanism of Haloxylon in China. Biodiversity Science, 30, 21192. doi: 10.17520/biods.2021192.

\section{Historical distribution pattern and driving mechanism of Haloxylon in China}

Dan Zhang ${ }^{1}$, Songmei Ma ${ }^{2 *}$, Bo Wei ${ }^{1}$, Chuncheng Wang ${ }^{1}$, Lin Zhang ${ }^{2}$, Han Yan $^{2}$

1 Construction Corps Key Laboratory of Oasis Town and Mountain-basin System Ecology, College of Life Sciences, Shihezi University, Shihezi, Xinjiang 832000

2 Construction Corps Key Laboratory of Oasis Town and Mountain-basin System Ecology/Key Laboratory of Landscape Ecology in Arid Areas, College of Sciences, Shihezi University, Shihezi, Xinjiang 832000

\begin{abstract}
Aims: Under the influence of Quaternary climate fluctuation, xerophytes may have experienced large-scale migration and expansion. It is highly important to study the historical and geographical distribution pattern of xerophytes to understand the evolution of xerophyte flora under the background of climate fluctuation. The purpose of this study is to reveal the distribution pattern and driving mechanisms of Haloxylon in China since the last interglacial period (LIG) and the last glacial maximum period (LGM).

Methods: Based on 85 natural distribution sites and 2 sets of environmental factors, the geographic distribution pattern change, and driving mechanisms of Haloxylon since the LIG were analyzed by integrating GIS spatial analysis and 9 species distribution models. Based on the chloroplast gene data of 62 Haloxylon populations, we used the least cost path
\end{abstract}

收稿日期: 2021-05-13; 接受日期: 2021-09-14

基金项目: 国家自然科学基金(41561007; 41261011)和草原生态修复治理补助项目(XJCYZZ202007)

* 通讯作者 Author for correspondence. E-mail: shzmsm@126.com 
method to simulate the possible diffusion paths of Haloxylon since the LIG. Principal component analysis (PCA) of environmental variables affecting the distribution of Haloxylon was carried out by using the prcomp function in R 4.1.1. The contribution of environmental variables to the suitable distribution of Haloxylon was evaluated, and the correlation between key variables and the degree of suitability was analyzed.

Results: We found that the precision of the integrated model was significantly improved compared with that of the single model. Since the LIG, the distribution of Haloxylon has undergone a significant decrease and post-glacial expansion. From the LIG to the LGM, Haloxylon ammodendron retreated westward from Junggar Basin and western Tarim Basin to the northwest and eastern margin of Junggar Basin and northwest margin of Tarim Basin. Haloxylon persicum retreated from the western end of Junggar Basin and Tarim Basin to the south of Junggar Basin. Since the LGM, H. ammodendron has expanded eastward, through northern Gansu and eastern region of the Qaidam basin to Alxa Desert in western Inner Mongolia. Haloxylon persicum has expanded northeast on a small scale, occupying the central and western part of Junggar Basin. The change of key climatic factors in the suitable distribution area of Haloxylon is relatively low. PCA results showed that the score coefficient of the precipitation factor in the first principal component is higher. The analysis of the relationship between the environmental factors affecting the distribution of Haloxylon and the suitability of the distribution area demonstrated that there is a significant correlation between temperature factors and the suitability of the distribution of Haloxylon.

Conclusion: The climate change in Quaternary caused the geographical distribution of Haloxylon to narrow and then subsequently expand. The main migration paths of Haloxylon are through the Junggar Basin and Hexi Corridor. Precipitation factors mainly affect the change of suitable distribution area of Haloxylon, while temperature factors mainly affect the degree of distribution suitability of Haloxylon.

Key words: Haloxylon; historical distribution pattern; post glacial diffusion path; last glacial maximum period; last interglacial period

古气候记录显示，第四纪气候发生了多次冰期 和间冰期的往复振荡(鹿化煜和郭正堂, 2015)，强 烈影响了植被的历史发展动态, 塑造了其现代分布 格局。例如, 在末次盛冰期, 部分北方树种可能在 南方避难所生长(Svenning et al, 2008); 第四纪气候 变化导致黄连木属(Pistacia) 植物历史分布迁移 (Kozhoridze et al, 2015); 末次盛冰期以来的气候变 化导致陀螺果(Melliodendron xylocarpum)适宜生境 大幅度降低(王璐等, 2018); 历史气候变化促使长 花马先蒿(Pedicularis longiflora)发生了大范围迁移 扩张事件(于海涁等, 2014)。此外, 冰期-间冰期的循 环往复也导致植物在寒冷的冰期退缩至生境适宜 的避难所, 冰后期又从避难所迁移扩散到其他适宜 地区(Soltis et al, 2006)。例如, 对青藏高原地区一些 高山植物的研究表明: 在末次盛冰期, 这些植物退 缩至高原东部或东南部的冰期避难所, 冰期后大规 模扩张到高原上(Meng et al, 2007); 荒漠植物鹰爪 柴(Convolvulus gortschakovii)在冰期后由准喝尔盆 地边缘的避难所扩张至阿拉善沙漠(Jia \& Zhang, 2021)。追溯干旱荒漠植物的迁移扩散历史动态, 对 了解第四纪以来旱生植物的进化与演化历史以及 旱生植物区系的发生、发展等均具有重要意义。

基于地理信息系统 (geographic information system, GIS), 将遗传和地理空间数据融合在一起, 可以将宏观景观特征与微观遗传过程联系起来, 以 揭示物种的进化历史(Manel et al, 2003; Manel \& Segelbacher, 2009)。近年来, 基于GIS的方法或数据 开发的一些强大工具在生物地理学的许多领域发 挥了重要作用, 尤其是预测物种分布的模型 (species distribution models, SDMs) (Richards et al, 2007; Schorr et al, 2012)和物种扩散迁移路径的模 拟与可视化方法。

我国分布的梭梭属(Haloxylon)植物仅包括荒漠 植物梭梭 (H. ammodendron) 和沙生植物白梭梭 (H. persicum) (郭泉水等, 2005a), 均是第三纪子遗植物, 以这两种植物为优势的荒漠植被占西北荒漠区的 $10 \%$ 。梭梭喜生于砾石沙漠、粘土沙漠、固定和半 固定沙地以及盐地(郭泉水等, 2005b), 白梭梭则喜 生于固定沙丘、半固定沙丘、流动沙丘及丘间沙地 (魏岩等, 2005)。梭梭和白梭梭是荒漠地区盐生、旱 生植物群落的建群种, 能很好地适应沙漠地区干旱 缺水的恶劣生境, 并覆盖地表, 在维护干旱地区生 态平衡方面起着不可替代的作用, 如防风固沙、改 善沙区气候条件、为荒漠动物提供栖息环境等(郭泉 水等, 2005a)。然而, 近半个世纪以来, 由于环境恶 化、人为砍伐和过度放牧造成大面积的梭梭属植物 
衰退甚至死亡, 严重威胁中国西北地区沙漠生态系 统生态功能的发挥(赵天获, 2006; 杨燕南, 2015 ${ }^{\circledR}$ )。

研究气候变化对梭梭属植物潜在分布区域的 影响不仅利于中国西北地区沙漠生态系统的恢复, 也有助于梭梭属植物的科学保护。但是, 过去气候 变化对中国梭梭属历史分布格局的影响, 特别是末 次间冰期以来梭梭属植物的种群动态及其扩散路 径尚不清楚, 成为深入理解中国梭梭属历史分布对 气候变化的响应机制的瓶颈。本文围绕上述科学问 题, 利用 $\mathrm{R}$ 软件的Biomod 2 程序包集成的 9 种物种分 布模型, 结合GIS工具, 分析了末次间冰期以来梭 梭属在我国西北地区的适宜分布、迁移通道及历史 气候变化对梭梭属分布格局的影响。旨在探讨: (1) 末次间冰期以来中国梭梭属的历史地理分布格局 及其变迁; (2)中国梭梭属的冰期避难所推测及扩散 路径分析; (3)影响中国梭梭属适宜地理分布的环境 驱动因素。

\section{1 数据和方法}

\section{1 分布数据来源与处理}

对中国梭梭属的自然分布区进行野外调查, 收 集到62个梭梭属的自然分布点; 通过网络爬虫检索 国家标本资源共享平台 (http://www.nsii.org.cn/ 2017/)、中国植物智平台(http://www.iplant.cn/) 以及 国家教学标本资源共享平台(http://mnh.scu.edu.cn/) 的记录并检索相关文献(付贵全等, 2016; 常红等, 2018), 收集到664条分布记录, 包括新疆甘家湖梭 梭林、内蒙古哈腾套海、内蒙古乌拉特和青海柴达 木梭梭林 4 个国家级自然保护区的分布点。通过 Google Earth确定详细地理坐标, 矫正分布点偏差。 基于R软件raster程序包(Street, 2020)进一步剔除距 离过近和重复的分布点, 最终获得 85 条梭梭属分布 记录用于模型的模拟, 其中梭梭 60 条、白梭梭 25 条。 叶绿体基因片段(trnS-trnG)变异的单倍型信息来自 本课题组的测序数据 ${ }^{2}$ 。

\section{2 环境数据来源与处理}

气候数据采用 WorldClim (http://www.worldclim.org)的基准时期(1970-2000年)、末次间冰期

(1) 杨燕南 (2015) 青海柴达木梭梭林国家级自然保护区景观保 护与恢复研究. 硕士学位论文, 中南林业科技大学, 长沙.

(2) 魏博 (2020) 荒漠植物梭梭居群的遗传特征及保护策略研 究. 硕士学位论文, 石河子大学, 石河子.
( 140-120 ka B.P.)、末次盛冰期( 21 ka B.P.)的19个 生物气候变量。模型模拟使用的中国省级行政区划 底图来源于国家基础地理信息系统网站 (http://www.ngcc.cn/ngcc/)。考虑空间环境变量对物 种的限制作用, 可通过降低变量的空间维度以避免 过拟合现象, 运用 R 软件Hmisc程序包中的 cor函数 对 19 个气候因子进行Pearson相关性分析, 剔除相 关性高( $>80 \%)$ 的变量(Parolo et al, 2008)。最终共有 13 个气候因子用于分析(表1)。

\section{3 模型构建与评估}

$\operatorname{Biomod} 2$ 是基于 $\mathrm{R}$ 软件开发的物种分布模型集 合平台，包含多种算法，该平台可选择不同模型类 别, 设置不同的初始条件、参数和限制性来达到最 好的预测效果, 目前在物种分布模型构建中被广泛 使用。本研究利用Biomod2程序包中的9个生态位模 型, 包括广义线性模型(generalized linear models, GLM)、广义相加模型(generalized additive models, GAM)、分类树分析(classification tree analysis, CTA)、人工神经网络(artificial neural networks, ANN)、推进式回归树(generalized boosted models, GBM)、最大熵(maximum entropy, MaxEnt)、随机森 林(random forests, RF)、柔性判别分析(flexible discriminant analysis, FDA)和表面分布区分室模型 (one rectilinear envelope similar to bioclim, SRE)对 梭梭和白梭梭的适宜分布区分别进行模拟。由于模 型构建时需要物种的分布数据和伪存在数据, 本研 究基于梭梭和白梭梭的分布点数据, 利用Biomod 2 程序包分别生成 1,000 个伪存在点数据用于模型模 拟。模型构建过程中，随机选取 $75 \%$ 的样点数据用 作模型训练, 剩余 $25 \%$ 的样点数据用于模型验证, 每个模型算法进行 10 次重复, 并设置训练数据集和 验证数据集的分布点数据和伪存在点数据比例恒 定。采用加权平均法将所有TSS (true skill statistics) 值 $\geq 0.75$ 的单个模型集成, 以 TSS值为权重构建组 合模型, 并将所有模型结果导入QGIS3.12转换为 TIFF格式(高蓓等, 2017)。为了方便模型模拟结果的 可视化表达, 本研究选择模型最大TSS值作为阈值 (朱妮, 2019), 将模型结果区分为“适生区”和“不适 生区”, 为进一步提升可视化效果, 利用ArcGIS空 间分析工具提取分布点在适宜分布图上的适应性, 并计算这些值的标准差 $\sigma$ 和平均值 $\mu$, 选择 $\sigma+\mu$ 作 
表1 影响梭梭和白梭梭分布的气候变量的主成分(PC)特征值

Table 1 Principal component (PC) eigenvalues of climate variables affecting the distribution of Haloxylon ammodendron and $H$. persicum

\begin{tabular}{|c|c|c|c|c|c|c|c|c|c|c|c|c|}
\hline \multirow{2}{*}{$\begin{array}{l}\text { 气候因子 } \\
\text { Climatic factor }\end{array}$} & \multicolumn{6}{|c|}{ 梭梭 Haloxylon ammodendron } & \multicolumn{6}{|c|}{ 白梭梭 Haloxylon persicum } \\
\hline & PC1 & $\mathrm{PC} 2$ & PC3 & $\mathrm{PC} 4$ & PC5 & PC6 & $\mathrm{PC} 1$ & PC2 & PC3 & PC4 & PC5 & PC6 \\
\hline 平均气温日较差 & -0.4010 & -0.023 & -0.4471 & 0.3015 & 0.7218 & -0.0155 & 0.0800 & 0.0560 & 0.6657 & 0.0289 & 0.3137 & 0.5855 \\
\hline $\begin{array}{l}\text { Mean diurnal range (Bio2) } \\
\text { 温度季节性 } \\
\text { Temperature seasonality (Bio4) }\end{array}$ & 0.3534 & 0.3672 & 0.3143 & 0.3992 & 0.1067 & -0.2803 & & & & & & \\
\hline $\begin{array}{l}\text { 最热月最高温 Max temperature of } \\
\text { warmest month (Bio5) }\end{array}$ & & & & & & & -0.4205 & -0.3078 & -0.2280 & -0.2558 & 0.3226 & 0.0864 \\
\hline $\begin{array}{l}\text { 最冷月最低温 Min temperature of } \\
\text { coldest month (Bio6) }\end{array}$ & & & & & & & -0.3240 & 0.0336 & -0.5535 & 0.0760 & 0.1049 & 0.4440 \\
\hline $\begin{array}{l}\text { 气温年较差 } \\
\text { Annual temperature range (Bio7) }\end{array}$ & & & & & & & -0.2233 & -0.4475 & 0.2756 & -0.4223 & 0.3220 & $-\mathbf{0 . 3 5 0 3}$ \\
\hline $\begin{array}{l}\text { 最湿季平均温 Mean temperature of } \\
\text { wettest quarter (Bio8) }\end{array}$ & -0.0631 & 0.6370 & 0.4028 & -0.0623 & 0.3398 & 0.4079 & & & & & & \\
\hline $\begin{array}{l}\text { 最干季平均温 Mean temperature of } \\
\text { driest quarter (Bio9) }\end{array}$ & & & & & & & -0.3546 & 0.2862 & 0.0772 & 0.6046 & 0.5461 & -0.2837 \\
\hline $\begin{array}{l}\text { 最冷季平均温 Mean temperature of } \\
\text { coldest quarter (Bio11) }\end{array}$ & -0.3831 & 0.2188 & 0.0367 & -0.7455 & 0.0309 & -0.1367 & & & & & & \\
\hline $\begin{array}{l}\text { 年均降水量 } \\
\text { Annual precipitation (Bio12) }\end{array}$ & & & & & & & 0.4753 & -0.1496 & -0.2554 & -0.0359 & 0.4549 & 0.3195 \\
\hline $\begin{array}{l}\text { 最湿月降水量 Precipitation of wettest } \\
\text { month (Bio13) }\end{array}$ & 0.0528 & $-\mathbf{0 . 5 8 0 0}$ & 0.6229 & -0.1540 & 0.4640 & -0.1687 & 0.4583 & 0.2152 & -0.2196 & -0.2281 & 0.4103 & -0.2874 \\
\hline 最干月降水量 & 0.4469 & -0.0836 & -0.1889 & -0.2130 & 0.1093 & 0.3556 & & & & & & \\
\hline Precipitation of driest month (Bio14) & & & & & & & & & & & & \\
\hline $\begin{array}{l}\text { 降水季节性变化 } \\
\text { Precipitation seasonality (Bio15) }\end{array}$ & -0.4064 & -0.2396 & 0.2714 & 0.2735 & -0.2429 & 0.6464 & -0.0183 & 0.5997 & -0.0048 & -0.3225 & 0.0936 & -0.1350 \\
\hline $\begin{array}{l}\text { 最冷季降水量 Precipitation of coldest } \\
\text { season (Bio19) }\end{array}$ & 0.4426 & -0.1004 & -0.2010 & -0.2152 & 0.2551 & 0.4042 & 0.3162 & -0.4364 & -0.0558 & 0.4762 & 0.0238 & -0.2155 \\
\hline
\end{tabular}

为阈值, 从“适生区”中划分出“高度适生区” (魏博 等, 2019)。

模型评价选择AUC (area under the curve)和TSS 两种应用最广泛的评价指标。AUC值域范围为(0-1), 大于 0.8 表示模型结果精确; TSS值域范围为 $[-1,1]$, 取值越接近 1 表示模型精度越高。

\section{4 扩散路径分析}

基于物种分布频率越高的点位具有更低的迁 移成本这个假设(Chan et al, 2011), 首先, 将物种分 布模型转换成物种生境阻力模型, 即利用ArcGIS重 分类工具将每个时期梭梭属组合模型的结果图层 进行新值取反转换成生境阻力模型 $(1-\mathrm{SDM})$; 其 次, 使用 SDMtoolbox 2.0 (http://www.sdmtoolbox. org/)进行最小成本路径(least-cost path method, LCP) 模拟, 即基于梭梭属cpDNA数据集具有共享单倍型 的点位之间的迁移路线, 使用梭梭属的阻力层为每 个样本区域创建成本距离栅格, 在成本距离栅格的 基础上, 在每对居群之间建立连接路径; 最后, 将 所有成对路径层进行ArcGIS栅格叠加分析, 获得并 可视化梭梭属的扩散路径(Chan et al, 2011)。

\section{5 环境变量分析}

利用ArcGIS提取梭梭属每个分布点篮选后的 环境因子数值及其在集成模型结果中的适宜程度 数值, 利用 $\mathrm{R}$ 软件prcomp函数对上述提取的环境变 量进行主成分分析(PCA), 以评估气候变量对梭梭 属适宜性的贡献, 并利用提取的适宜程度数值进行 分组, 评估气候变量与适生程度的相关性, 以红色 至绿色的渐变表示显著性从高到低(王华辰等, $2020)$ 。计算每个时期的 $\mathrm{PC} 1$ 评分, 利用 $\left|P C 1_{\text {基准 }}\right|-$ $\left|\mathrm{PC} 1_{\mathrm{LGM}}\right|$ 和 $\left|\mathrm{PC} 1_{\mathrm{LGM}}\right|-\left|\mathrm{PC} 1_{\mathrm{LIG}}\right|$ 标准化结果比较不同 时期研究区的气候变化, 低值表明气候相对稳定, 高值表明气候差异较大(Jiang et al, 2019), 利用 ArcGIS重分类工具对标准化结果进行可视化。

\section{2 结果}

\section{1 不同模型的预测精度及空间表现力}

本研究中, 9 种模型的模拟精度存在较大差异, 集成模型的精度及可视化结果较单一模型均显著 提升(图1, 图2)。其中, 随机森林模型的模拟精度最 高且重复建模的结果较稳定, 模拟结果的平均TSS 


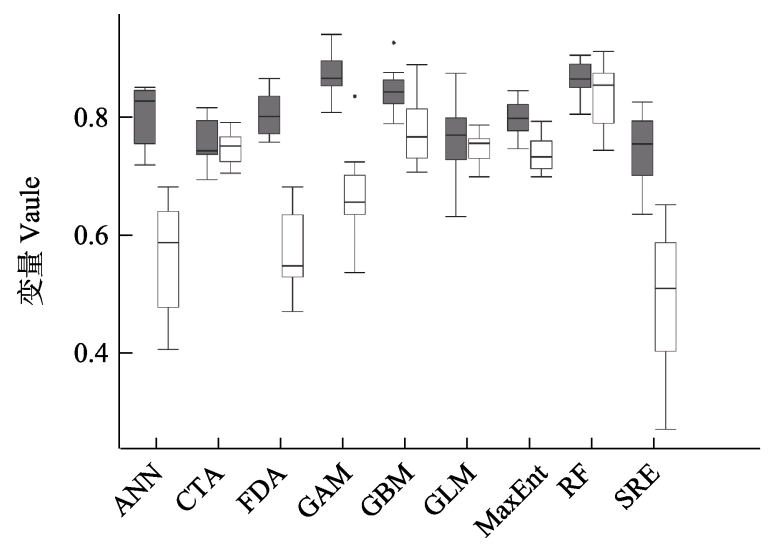

梭梭 H. ammodendron

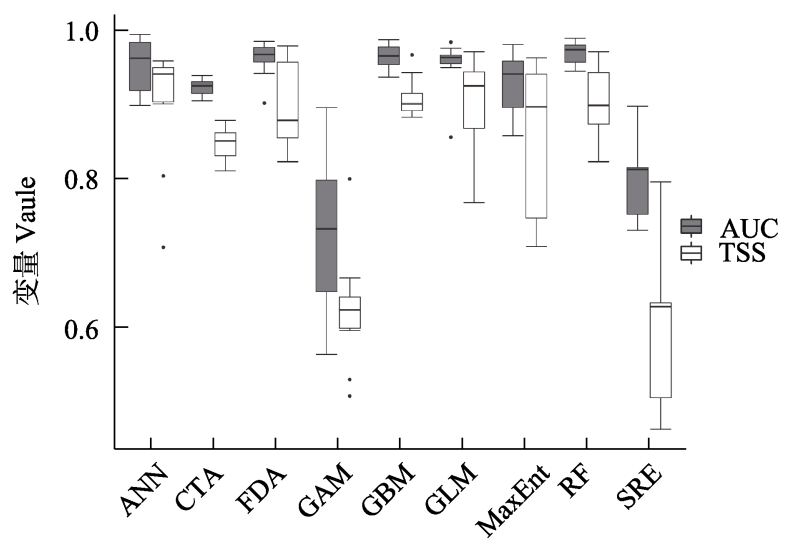

白梭梭 H. persicum

图1 不同模型预测精度评价。ANN：人工神经网络; CTA：分类树分析; FDA：柔性判别分析; GAM: 广义相加模型; GBM: 推进式回归树; GLM: 广义线性模型; MaxEnt: 最大熵模型; RF：随机森林; SRE：表面分布区分室模型; AUC: 特征曲线下 的面积; TSS: 真实技巧统计值。

Fig. 1 Evaluation of prediction results of different models. ANN, Artificial neural networks; CTA, Classification tree analysis; FDA, Flexible discriminant analysis; GAM, Generalized additive models; GBM, Generalized boosted models; GLM, Generalized linear models; MaxEnt, Maximum entropy; RF, Random forests; SRE, One rectilinear envelope similar to bioclim; AUC, Area under the curve; TSS, True skill statistics.
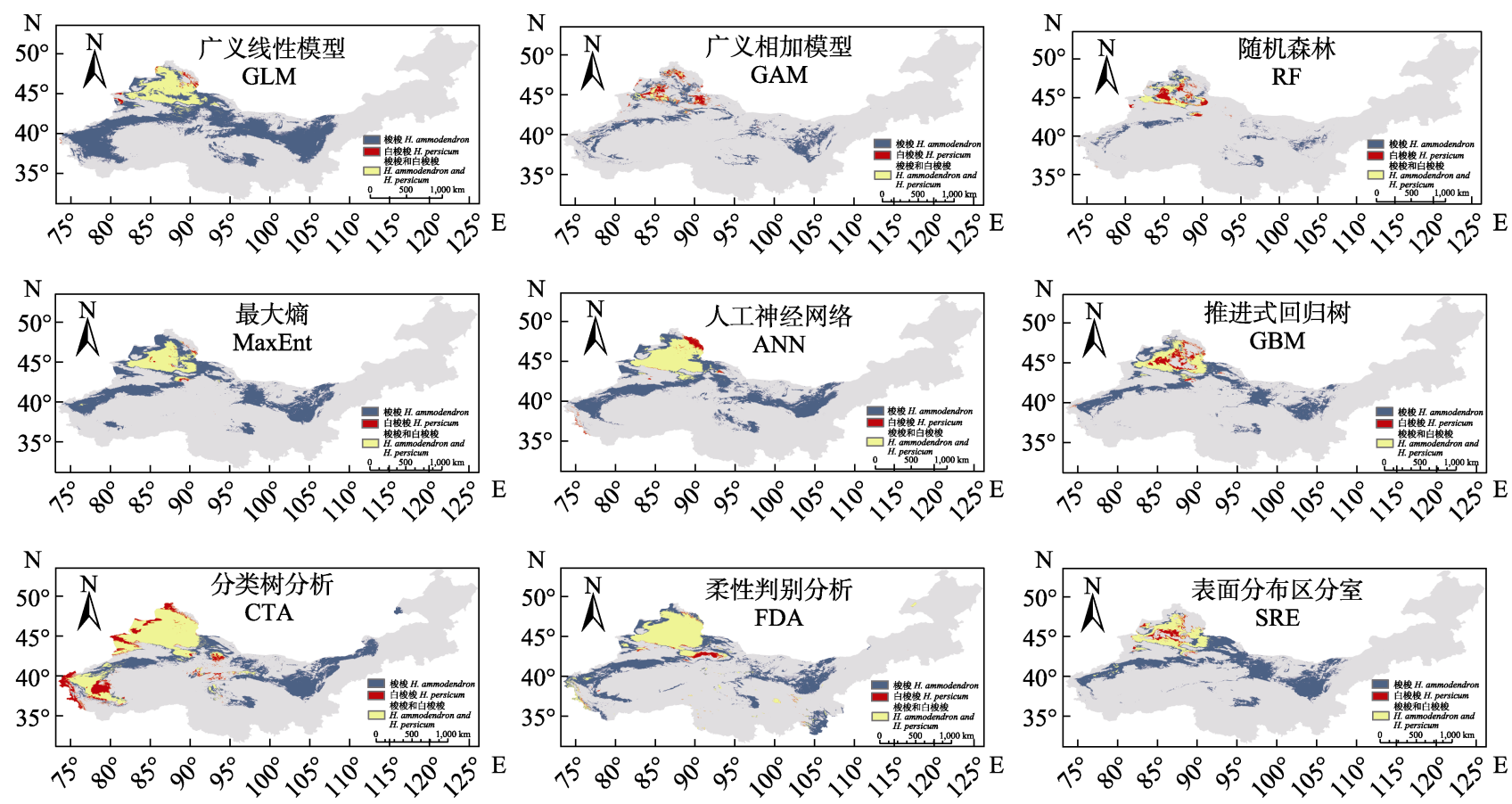

图2 基准气候(1970-2000年)条件下不同模型模拟的中国梭梭属植物的适宜分布

Fig. 2 Suitable distributions of Haloxylon plants in China simulated by different models in the base period (1970-2000). ANN, Artificial neural networks; CTA, Classification tree analysis; FDA, Flexible discriminant analysis; GAM, Generalized additive models; GBM, Generalized boosted models; GLM, Generalized linear models; MaxEnt, Maximum entropy; RF, Random forests; SRE, One rectilinear envelope similar to bioclim.

和AUC均高于 0.8 。而表面分布区分室模型和广义加 法模型的表现最差, 平均 TSS 和 AUC均低于 0.74 , 且检验结果不稳定, 不符合模型检验的最低标准,
因此未参与集成模型的构建。其他6种模型的模拟 精度介于上述3种模型之间, 最终梭梭的集成模型 结果由 5 个单一模型构成(ANN、GAM、GLM、 
MaxEnt和RF)，白梭梭的集成模型结果由 7 个单一 模型构成(ANN、CTA、FDA、GBM、GLM、MaxEnt 和RF, 图1)。此外, 无论集成模型还是单一模型, 对 梭梭和白梭梭及其不同时期的模拟结果均存在一 定差异, 白梭梭的模拟精度总体均高于梭梭。

\section{2 中国梭梭属植物历史地理分布格局}

集成模型的模拟结果表明, 3 个时期梭梭属植 物均在准噶尔盆地和塔里木盆地存在小面积的分 布, 且分布范围均经历了冰期收缩和冰后期再扩张 (图3)。梭梭在末次间冰期占据了整个准噶尔盆地及 塔里木盆地西部; 至末次盛冰期时大面积向西退缩 至准噶尔盆地的西北缘、东缘以及塔里木盆地的西
北缘; 末次盛冰期至今, 又大面积向东沿甘肃北 部、柴达木盆地东部扩张至内蒙古西部阿拉善荒 漠。然而，白梭梭在末次间冰期仅分布于准噶尔盆 地以及塔里木盆地西端; 至末次盛冰期向西南方向 退缩, 仅存在于准噶尔盆地南缘; 末次盛冰期至今, 仅向东北方向小范围扩张，在准噶尔盆地的中西部 有分布(图3)。

扩散路径分析结果表明，末次间冰期以来中国 梭梭属植物显示出相似的沿沙漠、戈壁迁移的扩散 模式, 准噶尔盆地和河西走廊是梭梭最重要的扩散 路径, 其中, 准噶尔盆地成为连接梭梭南北居群的 重要路径, 河西走廊成为连接东西居群的重要路

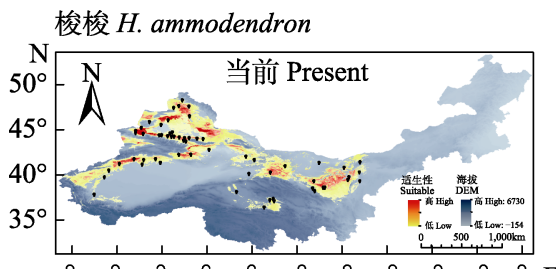

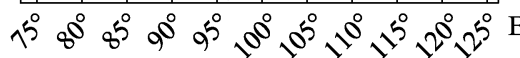

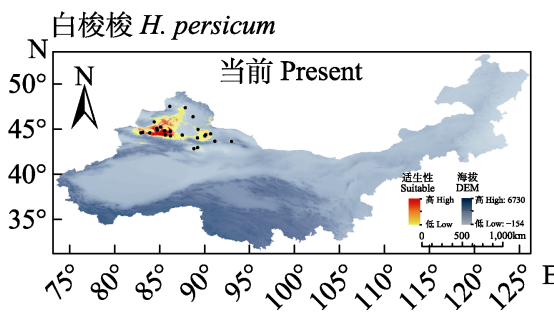

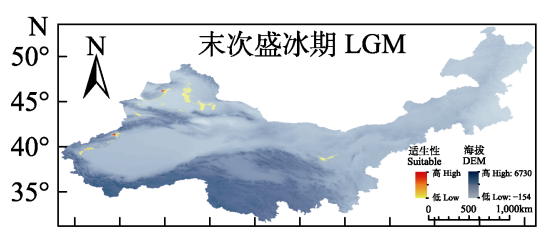

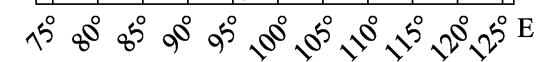
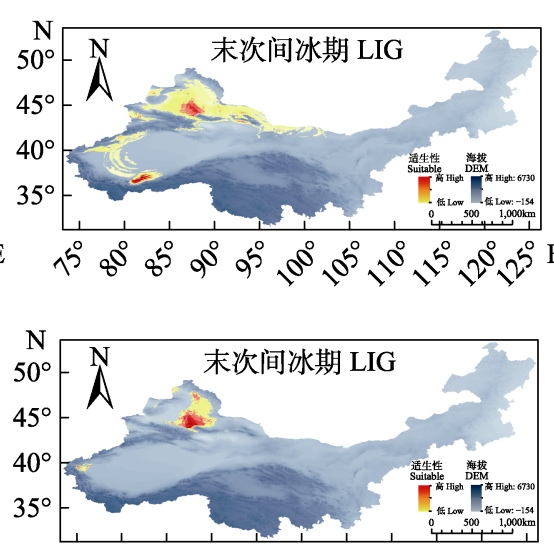

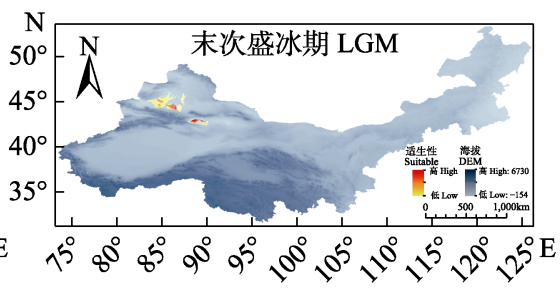

$15^{\circ} 8^{\circ} 5^{\circ}, 9^{\circ}, 9^{\circ}, 0^{\circ}, 0^{\circ}, 12^{\circ}, 5^{\circ}, 2^{\circ}, 2^{\circ} \mathrm{E}$

图3中国梭梭属植物不同时期的适宜分布

Fig. 3 Suitable distribution of Haloxylon in different periods in China. LGM, Last glacial maximum period; LIG, Last interglacial period.

梭梭 H. ammodendron

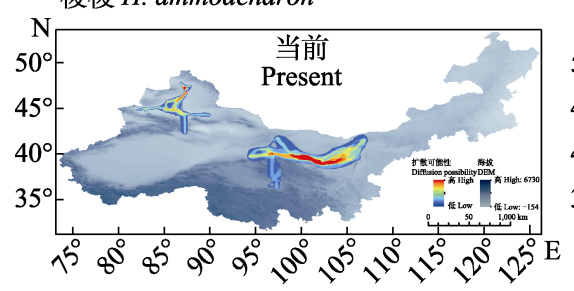

白梭梭 H. persicum

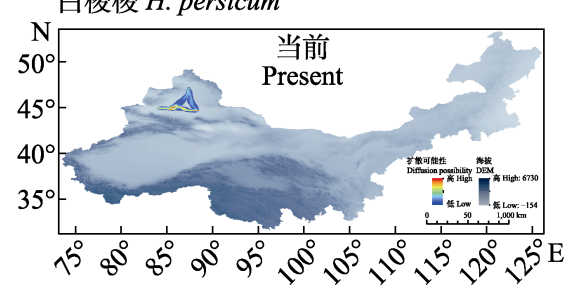

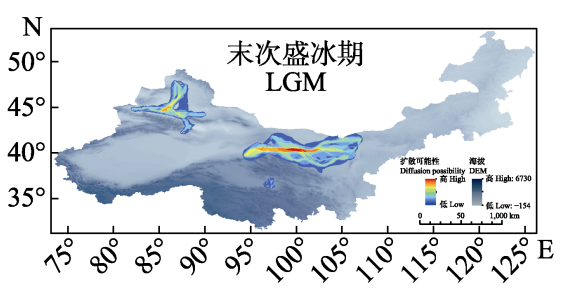
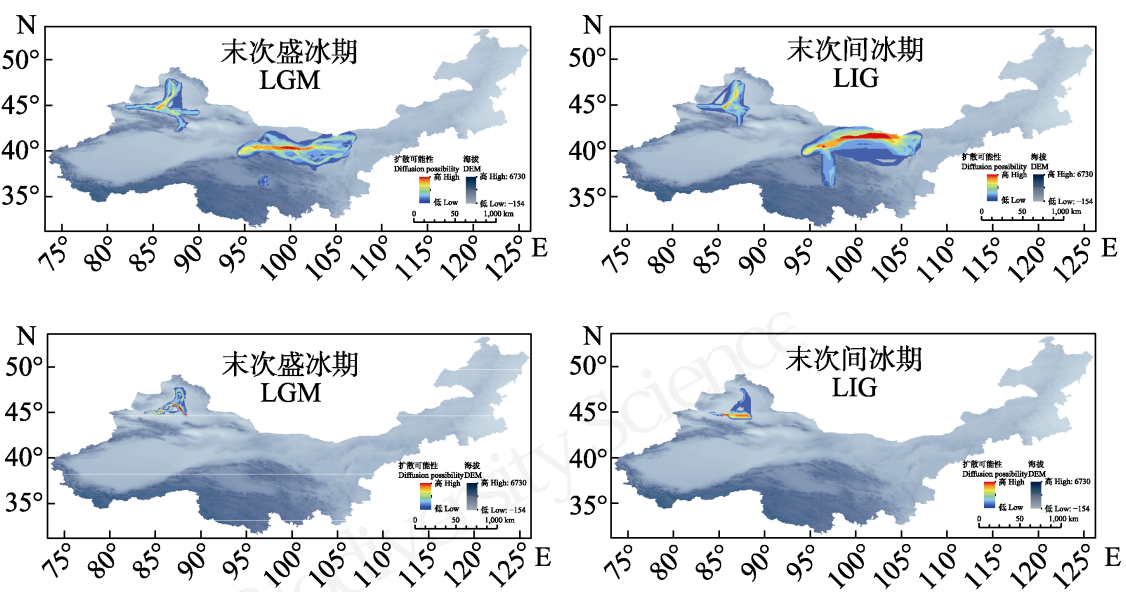

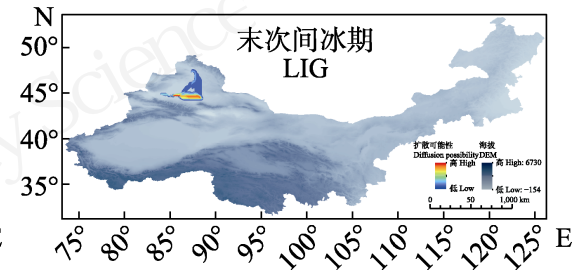

图4 中国梭梭属不同时期可能的迁移扩散路径

Fig. 4 Possible migration and diffusion paths of Haloxylon in different periods in China. LGM, Last glacial maximum period; LIG, Last interglacial period. 
径。古尔班通古特沙漠则是连接白梭梭东西部居群 的最重要的扩散路径(图4)。

\section{3 影响梭梭属适宜分布的关键驱动因子及其异} 质性

基准气候因子的主成分分析结果表明，降水因 子对梭梭属分布的影响较大。其中, 梭梭的前两个 主成分解释了环境因子对 $83.9 \%$ (PC1: 57.9\%; PC2: $26.0 \%)$ 的分布点的影响，第一主成分中降水因子的 得分系数较高, 而第二主成分中温度因子的得分较

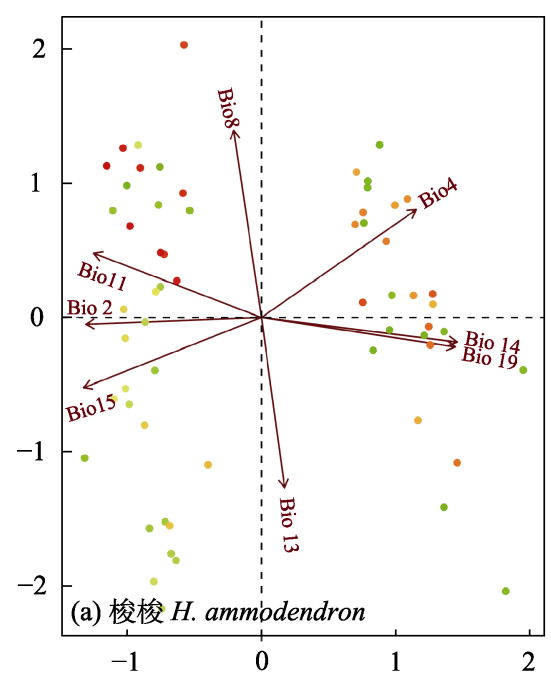

高; 白梭梭的前两个主成分解释了环境因子对 64.1\% (PC1: 36.2\%; PC2: 27.9\%)的分布点的影响, 前两个主成分均显示出降水因子的得分较高(表1, 图5)。影响梭梭属分布的环境因子与分布区适宜性 程度关系的分析表明, 温度因子与梭梭属分布适宜 性的高低呈显著相关, 最湿季平均温和最冷季平均 温与梭梭适宜性程度呈显著正相关; 最冷月最低温 和最干季平均温与白梭梭的分布适宜性呈显著正 相关。

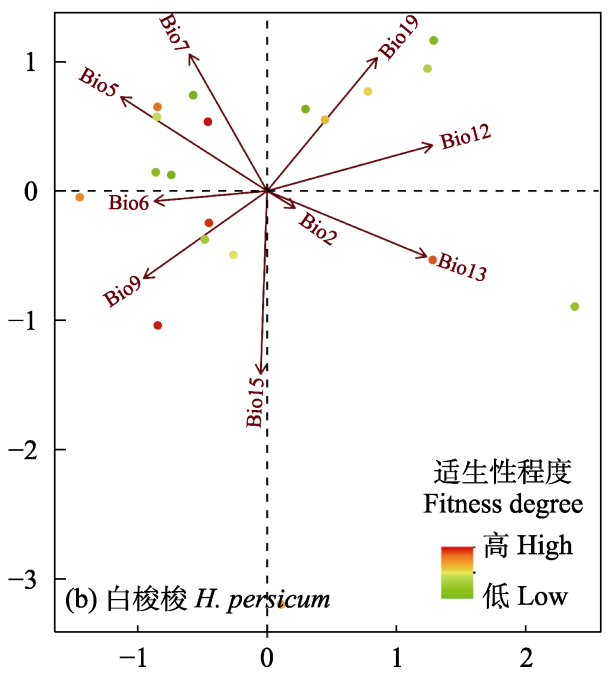

图5 基准气候(1970-2000年)条件下影响梭梭属植物分布的气候因子的主成分分析(PCA)。图中气候变量名称见表1。

Fig. 5 Principal component analysis (PCA) of climatic factors affecting the distribution of Haloxylon under baseline climate (1970-2000). The names of climate variables in the figure are shown in Table 1.
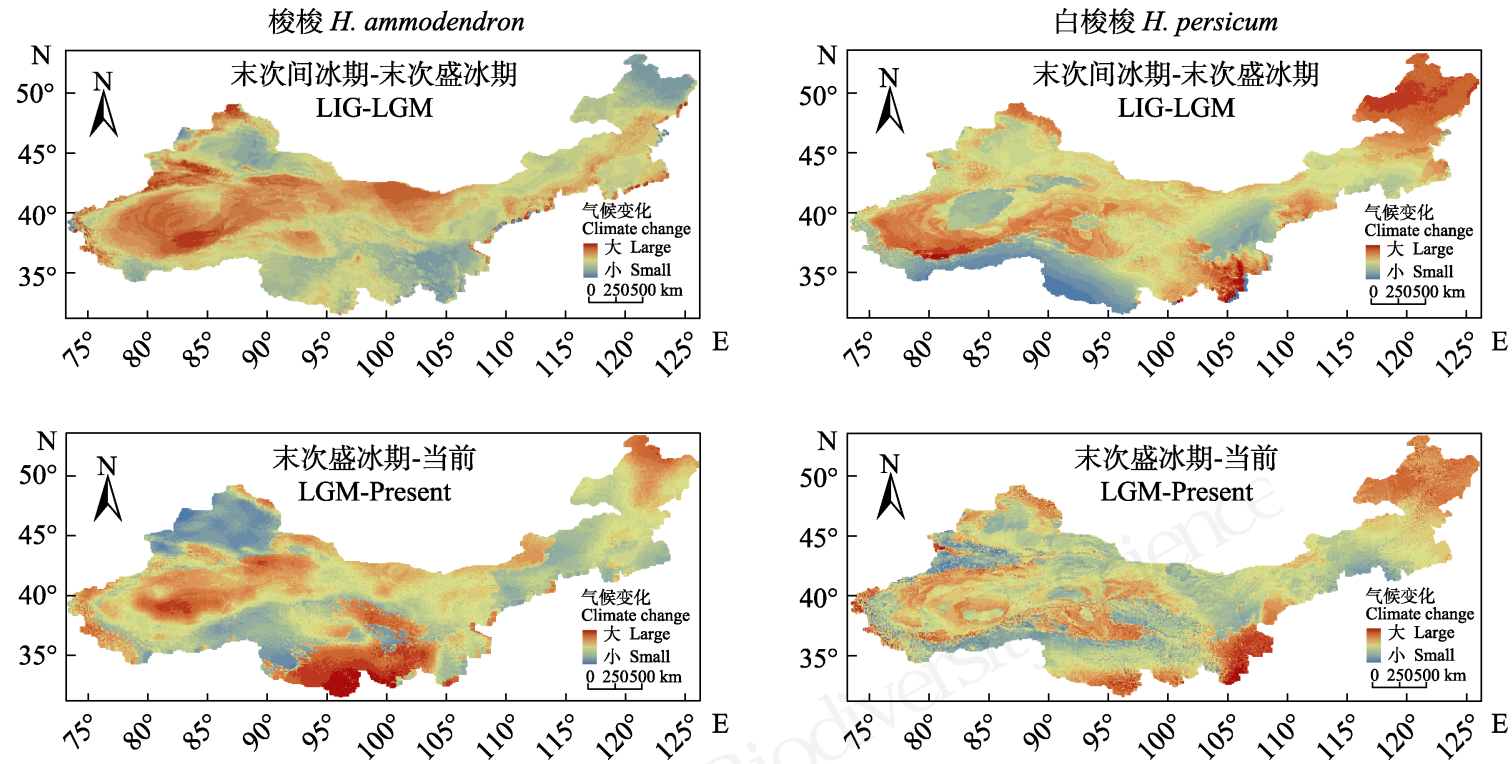

图6 不同时段影响梭梭和白梭梭分布的气候因子的变化程度

Fig. 6 Changes of climatic factors affecting the distribution of Haloxylon ammodendron and H. persicum in different periods. LGM, Last glacial maximum period; LIG, Last interglacial period. 
研究区的气候差异显示了不同时期气候因子 的变化幅度(图6)。可以看出, 梭梭属植物的适生区 域均分布于气候波动相对较低的区域, 如准噶尔盆 地、塔里木盆地东北部和河西走廊西部等, 表明间 冰期以来的气候波动对梭梭属植物的分布存在较 大限制。具体来说，末次间冰期至末次盛冰期，准 噶尔盆地南部、塔里木盆地西北部的气候波动较显 著, 同时梭梭属的分布面积也大幅度收缩至准噶尔 盆地中部; 而末次盛冰期至今, 研究区北部的干旱 地区的气候波动较小, 如准噶尔盆地、甘肃北部、 柴达木盆地以及内蒙古西部的阿拉善荒漠等, 梭梭 属植物的分布也沿这些戈壁和沙漠的边缘扩张。

\section{3 讨论}

本研究结果表明, 集成模型在模拟精度及可视 化效果方面较单一模型均有所提升(图1, 图2), 在 人参(Panax ginseng) (赵泽芳等, 2016)、雪落樱桃 (Cerasus xueluoensis) (王华辰等, 2020)的潜在分布 模拟研究中也得到了相似的结果, 这是因为单一模 型算法很难在所有情况下都能够得到最优结果, 集 合模型则可以降低单一模型的不确定性, 进而显著 提高物种分布预测的准确性 ${ }^{(1)}$ 。相关研究表明, 物种 分布的广度、聚散模式等显著影响模型模拟的准确 性(Marmion et al, 2009), 本文中模型对白梭梭的模 拟精度显著高于梭梭, 这是因为梭梭的分布范围较 白梭梭更广, 在分布的聚散模式上也更为破碎, 因 此在模型识别模拟时更为复杂。

本研究中, 第四纪以来中国梭梭属植物的历史 分布均经历了先收缩后扩张(图3)。利用系统地理学 方法对旱生植物的研究表明, 自末次间冰期以来麻 黄属(Ephedra)、白刺属(Nitraria)植物也经历了类似 的历史分布变迁(刘运东等, 2009; Yin et al, 2020)。 这可能是由于第四纪冰期的气候并不是持续寒冷, 而是从年际到千年尺度的明显的气候波动, 末次间 冰期表现为温暖干旱气候(刘显, 2008), 较适宜类 似梭梭属的荒漠植物生长, 因而梭梭属植物能够广 泛分布于我国西北地区。而末次盛冰期, 青藏高原 的隆升及季风的影响导致西北地区冷寒气候的形 成, 对植物造成了极大的影响, 大多数植物在冰期

(1) 孔晓泉 (2015) 物种分布模型的稳定性评估及应用软件. 硕 士学位论文, 安庆师范学院, 芜湖.
的分布范围大幅减小，甚至消失、濒临灭绝，梭梭 属植物也可能受气候的影响经历了大范围的分布 收缩。

此外, 本研究结果表明, 梭梭和白梭梭表现出 相似的沿沙漠、盆地边缘扩散迁移的特点(图4)。梭 梭和白梭梭为同域分布植物, 两种植物间的生态位 分化并不显著(孙芳芳等, 2019), 因此可能存在较 相似的进化历史。在冰后期, 随着气候逐渐回暖, 干旱的环境引发了广泛的沙漠发育, 西北地区很多 荒漠植物表现出沿沙漠、戈壁大规模种群扩张现象, 但物种的扩张并不是盲目和无序的, 都会遵循最低 迁移成本的原则进行, 通常会沿着河道、沟谷、平 地等平缓地形进行迁移(于海樹等, 2014)。因此, 西 北地区广泛分布的盆地、沙漠成为梭梭属植物迁移 扩张的最佳通道, 且气候波动结果显示, 不同时期 研究区的盆地、沙漠区域相对具有较小的气候差异, 更适宜梭梭和白梭梭的迁移(图6)。然而, 梭梭的扩 散路径并不连续, 而是存在明显的间断式分布，这 可能是由于第四纪冰期以来, 新疆东部及甘肃西部 气候波动较大, 且分布着祁连山、阿尔金山等山脉, 限制了梭梭属植物的扩散, 导致扩散路径分化。本 研究结果还表明, 自末次间冰期以来, 梭梭属植物 均在准噶尔盆地、塔里木盆地边缘存在小面积的高 度适生的分布斑块, 推测很可能是梭梭属植物的多 个分离的冰期避难所。基于孢粉数据的研究也表明, 第三纪期间准噶尔盆地及塔里木盆地存在梭梭属 植物(宋之琛等, 2008)。准噶尔盆地的梭梭属居群也 存在较高的遗传多样性(Suo et al, 2012)。经历气候 的极端变化后, 部分植物在漫长的末次盛冰期退缩 至相对适宜的区域, 并在气候条件适宜时向外扩展 栖息地，这些适宜的区域即成为了存活植物的冰期 避难所。

气候变化对物种的分布甚至存亡具有重要的 影响, 物种历史分布格局变迁通常又在一定程度上 反映了气候的波动特征。本研究表明, 相对冰后期 的气候变暖, 冰期的大幅度降温对梭梭属植物分布 格局的影响较大，导致其分布面积急速缩减(图3)。 由于冰期温度的大幅下降，青藏高原从第三纪开始 的长时间连续升高, 阻碍了来自印度洋的潮湿海风, 导致中国西北部出现干旱气候, 从而形成西北地区 复杂的地貌和干旱的气候, 随着干旱化向内蒙古的 
扩展, 中新世晚期至上新世, 硬叶植物和旱生植物 才逐渐形成 。因此, 相对冰后期的逐渐回暖, 末次 盛冰期气温的大幅度下降对梭梭属植物的分布格 局影响更大。本研究结果还表明, 降水和温度共同 制约着梭梭属植物的分布, 其中降水主导着分布面 积的变化, 而温度则与其适应性高低显著相关(表 1 , 图5)。水分在干旱区是影响植物存活、生长的主要 限制因子。近几十年的研究表明, 即使梭梭和白梭 梭能够分别通过不同的水分利用策略合理地利用 干旱区有限的水资源以维持自身的生长发育, 但由 于在干旱季节气温上升加快且波动剧烈, 导致大气 干旱加剧, 引起浅层土壤水分丢失, 使得梭梭属幼 苗大量死亡, 进而影响成年梭梭属植株的存活、繁 殖, 制约了梭梭属种群的分布和更新。此外, 利用 遥感技术对西北地区梭梭属植物的研究也表明, 梭 梭属群落的盖度和生物量与生长季温度的偏相关 最高(常静等, 2006)。冰期后, 西北地区的气温显著 回升, 尤其是近几十年来, 春季气候急剧变暖, 这 种强烈的变暖增加了沙漠生态系统的蒸散量, 也导 致梭梭植被大面积退化(Zhao et al, 2021)。

\section{参考文献}

Chan LM, Brown JL, Yoder AD (2011) Integrating statistical genetic and geospatial methods brings new power to phylogeography. Molecular Phylogenetics and Evolution, 59, 523-537.

Chang H, Liu T, Liu HF, Du HY (2018) The impacts of climate change on the potential habitat of Haloxylon anmodendron and uncertainty analysis. Journal of Shihezi University (Natural Science), 36, 351-357. (in Chinese with English abstract) [常红, 刘䑣, 刘华峰, 杜皓阳 (2018) 气候变化 对我国梭梭潜在分布的影响及不确定性分析. 石河子大 学学报(自然科学版), 36, 351-357.]

Chang J, Pan CD, Shi RF (2006) Analysis on dominant species distribution patterns and relation of Ass. Haloxylon persicum $+H$. ammodendron. Journal of Xinjiang Agricultural University, 29(2), 26-29. (in Chinese with English abstract) [常静, 潘存德, 师瑞锋 (2006) 梭梭 白梭梭群落优势种种群分布格局及其种间关系分析. 新 疆农业大学学报, 29(2), 26-29.]

Fu GQ, Xu XY, Ma JP, Xu MS, Liu J, Ding AQ (2016) Responses of Haloxylon ammodendron potential geographical distribution to the hydrothermal conditions under MaxEnt model. Pratacultural Science, 33, 2173-2179. (in Chinese with English abstract) [付贵全, 徐先英, 马剑

(1) 应红 (2016) 柴达木盆地东北缘中中新世以来气候与化学风 化作用的演化. 硕士学位论文, 兰州大学, 兰州.
平，徐梦莎，刘江，丁爱强 (2016) 基于MaxEnt下梭梭潜 在地理分布对水热条件的响应. 草业科学, 33, 21732179.]

Gao B, Hu N, Guo YL, Gu W, Zou JY (2017) Comparison of the potential geographical distribution of foxtail millet (Setaria italica) predicted by different models. Chinese Journal of Applied Ecology, 28, 3331-3340. (in Chinese with English abstract) [高蓓, 胡凝, 郭彦龙, 顾蔚, 邹继 业 (2017) 中国谷子潜在地理分布的多模型比较. 应用 生态学报, 28, 3331-3340.]

Guo QS, Guo ZH, Yan H, Wang CL, Tan DY, Ma C, He HY (2005a) Study on potential distribution of Haloxylon plants dominated desert vegetation in China. Acta Ecologica Sinica, 25, 848-853, 946. (in Chinese with English abstract) [郭泉水, 郭志华, 阎洪, 王春玲, 谭德远, 马超, 何红艳 (2005a) 我国以梭梭属植物为优势的潜在荒漠植被分布. 生态学报, 25, 848-853, 946.]

Guo QS, Wang CL, Guo ZH, Tan DY, Shi ZM (2005b) Geographic distribution of existing Haloxylon desert vegetation and its patch character in China. Scientia Silvae Sinicae, 41(5), 2-7, 219. (in Chinese with English abstract) [郭泉水, 王春玲, 郭志华, 谭德远, 史作民 (2005b) 我 国现存梭梭荒漠植被地理分布及其斑块特征. 林业科学, 41(5), 2-7, 219.]

Jia SW, Zhang ML (2021) Introgression of phylogeography lineages of Convolvulus gortschakovii (Convolvulaceae) in the northwest China. Plant Systematics and Evolution, 307, doi: 10.1007/s00606-020-01734-z.

Jiang XL, An M, Zheng SS, Deng M, Su ZH (2019) Geographical isolation and environmental heterogeneity contribute to the spatial genetic patterns of Quercus kerrii (Fagaceae). Heredity, 120, 219-233.

Kozhoridze G, Orlovsky N, Orlovsky L, Blumberg DG, Golan-Goldhirsh A (2015) Geographic distribution and migration pathways of Pistacia-Present, past and future. Ecography, 38, 1141-1154.

Liu Y (2008) Simulations of Climate Changes over China in LGM and Mid-Holocene. PhD dissertation, Nanjing University of Information Science \& Technology, Nanjing. (in Chinese with English abstract) [刘显 (2008) 末次冰期 冰盛期和中全新世中国地区气候变化的数值研究. 博士 学位论文, 南京信息工程大学, 南京.]

Liu YD, Qi YT, Qiu YJ, Zhang H, Wang SM (2009) The geographical distribution, origin and evolution of Ephedra. Journal of Arid Land Resources and Environment, 23, 120-126. (in Chinese with English abstract) [刘运东, 齐妍 婷, 邱远金, 张浩, 王绍明 (2009) 麻黄属的地理分布与 起源演化. 干旱区资源与环境, 23, 120-126.]

Lu HY, Guo ZT (2015) Impact of climatic change and human activity on desert and sand field in northern china since the last glacial maximum. China Basic Science, 17(2), 3-8. (in Chinese with English abstract) [鹿化显, 郭正堂 (2015) 末 次盛冰期以来气候变化和人类活动对我国沙漠和沙地环 境的影响. 中国基础科学, 17(2), 3-8.]

Manel S, Schwartz MK, Luikart G, Taberlet P (2003) Landscape genetics: Combining landscape ecology and population 
genetics. Trends in Ecology \& Evolution, 18, 189-197.

Manel S, Segelbacher G (2009) Perspectives and challenges in landscape genetics. Molecular Ecology, 18, 1821-1822.

Marmion M, Luoto M, Heikkinen RK, Thuiller W (2009) The performance of state-of-the-art modelling techniques depends on geographical distribution of species. Ecological Modelling, 220, 3512-3520.

Meng LH, Yang R, Abbott RJ, Miehe G, Hu TH, Liu JQ (2007) Mitochondrial and chloroplast phylogeography of Picea crassifolia Kom. (Pinaceae) in the Qinghai-Tibetan Plateau and adjacent highlands. Molecular Ecology, 16, 4128-4137.

Parolo G, Rossi G, Ferrarini A (2008) Toward improved species niche modelling: Arnica montana in the Alps as a case study. Journal of Applied Ecology, 45, 1410-1418.

Richards CL, Carstens BC, Lacey Knowles L (2007) Distribution modelling and statistical phylogeography: An integrative framework for generating and testing alternative biogeographical hypotheses. Journal of Biogeography, 34, 1833-1845.

Schorr G, Holstein N, Pearman PB, Guisan A, Kadereit JW (2012) Integrating species distribution models (SDMs) and phylogeography for two species of alpine Primula. Ecology and Evolution, 2, 1260-1277.

Soltis DE, Morris AB, McLachlan JS., Manos PS, Soltis PS. (2006) Comparative phylogeography of unglaciated eastern North America. Molecular Ecology, 15, 4261-4293.

Song ZC, Wang WM, Mao FY (2008) Palynological implications for relationship between aridification and monsoon climate in the tertiary of NW China. Acta Palaeontologica Sinica, 47, 265-272. (in Chinese with English abstract) [宋之琛, 王伟铭, 毛方园 (2008) 依据狍粉资料 探讨我国西北地区第三纪时期的干旱化及其与季风的关 系. 古生物学报, 47, 265-272.]

Street GM (2020) Habitat suitability and distribution models with applications in R. The Journal of Wildlife Management, 84, 1212-1213.

Sun FF, Nie YB, Ma SM, Wei B, Ji WQ (2019) Species differentiation of Haloxylon ammodendron and Haloxylon persicum based on ITS and cpDNA sequences. Scientia Silvae Sinicae, 55(3), 43-53. (in Chinese with English abstract) [孙芳芳, 聂迎涁, 马松梅, 魏博, 吉万全 (2019) 基于ITS和 cpDNA序列的梭梭和白梭梭物种分化. 林业 科学, 55(3), 43-53.]

Suo ZL, Jia ZQ, Lu Q, Pan BR, Jin XB, Xu G, Peng XQ, Sun HB, Tao YH (2012) Distinguishing Haloxylon persicum and H. ammodendron (Haloxylon Bunge, Amaranthaceae) using DNA marker. AASRI Procedia, 1, 305-310.

Svenning JC, Normand S, Kageyama M (2008) Glacial refugia of temperate trees in Europe: Insights from species distribution modelling. Journal of Ecology, 96, 1117-1127.

Wang HC, Zhu H, Li YF, Yi XG, Li M, Nan CH, Wang XR (2020) Potential distribution and ecological characteristic of Chinese endemic species Cerasus xueluoensis. Journal of Tropical and Subtropical Botany, 28, 136-144. (in Chinese with English abstract) [王华辰, 朱弘, 李涌福, 伊贤贵, 李蒙, 南程慧, 王贤荣 (2020) 中国特有植物雪落樱桃潜
在分布及其生态特征. 热带亚热带植物学报, 28 , 136-144.]

Wang L, Xu XG, Li Y (2018) Prediction of potential geographical distribution pattern change for Melliodendron xylocarpum Handel-Mazzetti since the Last Glacial Maximum. Chinese Journal of Ecology, 37, 278-286. (in Chinese with English abstract) [王璐, 许晓岗, 李圭 (2018) 末次盛冰 期以来陀螺果潜在地理分布格局变迁预测. 生态学杂志, 37, 278-286.]

Wei B, Ma SM, Song J, He LY, Li XC (2019) Prediction of the potential distribution and ecological suitability of Fritillaria walujewii. Acta Ecologica Sinica, 39, 228-234. (in Chinese with English abstract) [魏博, 马松梅, 宋佳, 贺凌云, 李 晓辰 (2019) 新疆贝母潜在分布区域及生态适宜性预测. 生态学报, 39, 228-234.]

Wei Y, Yin LK, Yan C (2005) Study on the flowering and wind-borne pollination characteristics of Haloxylon persicum. Arid Zone Research, 22, 85-89. (in Chinese with English abstract) [魏岩, 尹林克, 严成 (2005) 白梭梭开 花及风媒传粉特点. 干旱区研究, 22, 85-89.]

Yin HX, Wang LR, Shi Y, Qian CJ, Zhou HK, Wang WY, Ma XF, Tran LP, Zhang BY (2020) The East Asian winter monsoon scts as a major selective factor in the intraspecific differentiation of drought-tolerant Nitraria tangutorum in Northwest China. Plants (Basel), 9, 1100.

Yu HB, Zhang YL, Li SC, Qi W, Hu ZJ (2014) Predicting the dispersal routes of alpine plant Pedicularis longiflora (Orobanchaceae) based on GIS and species distribution models. Chinese Journal of Applied Ecology, 25, 16691673. (in Chinese with English abstract) [于海涁, 张镱锂, 李士成, 祁威, 胡忠俊 (2014) 基于GIS和物种分布模型 的高山植物长花马先蒿迁移路线模拟. 应用生态学报, 25, 1669-1673.]

Zhao L, Li WJ, Yang G, Yan K, He XL, Li FD, Gao YL, Tian LJ (2021) Moisture, temperature, and salinity of a typical desert plant (Haloxylon ammodendron) in an arid oasis of Northwest China. In: Sustainability, 13, 1908.

Zhao TB (2006) Studies on Spatial Distribution Pattern, Habitats Selection and Dynamic of Rhombomys opimus Population. $\mathrm{PhD}$ dissertation, Inner Mongolia University, Hohhot. (in Chinese with English abstract) [赵天䔸 (2006) 大沙鼠种群空间分布格局、栖息地选择及种群动态的研 究. 博士学位论文, 内蒙古大学, 呼和浩特.]

Zhao ZF, Wei HY, Guo YL, Gu W (2016) Potential distribution of Panax ginseng and its predicted responses to climate change. Chinese Journal of Applied Ecology, 27, 36073615. (in Chinese with English abstract) [赵泽芳, 卫海燕, 郭彦龙, 顾蔚 (2016) 人参潜在地理分布以及气候变化 对其影响预测. 应用生态学报, 27, 3607-3615.]

Zhu N (2019) Modelling the suitable habitat distribution of Magnolia officinalis using ensemble model. Journal of Sichuan Agricultural University, 37, 481-489. (in Chinese with English abstract) [朱妮 (2019) 基于组合物种分布模 型(ensemble model)的厚朴适宜生境分布模拟. 四川农业 大学学报, 37, 481-489.]

(责任编委：陈圣宾 责任编辑：黄祥忠) 